\title{
Predictors of postoperative urinary retention after semiclosed hemorrhoidectomy
}

\author{
Hong Yoon Jeong, Seok Gyu Song, Jong Kyun Lee \\ Department of Surgery, Seoul Songdo Hospital, Seoul, Korea
}

Purpose: This study was performed to analyze the predictors that might contribute to urinary retention following semiclosed hemorrhoidectomy under spinal anesthesia.

Methods: This retrospective study enrolled 2,176 consecutive patients with symptomatic grade III to IV hemorrhoids who underwent semiclosed hemorrhoidectomy between September 2018 and September 2019.

Results: Among the 2,176 patients, 1,878 (86.3\%) had no postoperative urinary retention, whereas 298 (13.7\%) developed urinary retention after hemorrhoidectomy. The percentage of males was significantly higher in the retention group than in the non-retention group $(60.4 \%$ vs. $48.1 \% ; \mathrm{P}=0.001)$. The risk of urinary retention was 1.52 -fold higher in males than in females (95\% confidence interval [CI], 1.13-2.04; $\mathrm{P}=0.005)$, 1.62-fold higher in old age (95\% CI, 1.14-2.28; $\mathrm{P}=0.006$ ), and 1.37 -fold higher with high body mass index (BMI) $(95 \% \mathrm{CI}, 1.04-1.81 ; \mathrm{P}=0.025)$. Patients with $\geq 4$ resected hemorrhoids had a higher odds ratio $(\mathrm{OR})$ of $1.46(95 \% \mathrm{CI}, 1.12-1.89 ; \mathrm{P}=0.005)$ than patients with < 4 resected hemorrhoids. Among the supplementary medication, patients who used analgesics had a higher OR of $2.06(95 \% \mathrm{CI}, 1.57-2.68 ; \mathrm{P}=0.001)$ than those who did not.

Conclusion: Male sex, age, high BMI, number of resected hemorrhoids, and supplementary analgesics are independent risk factors for urinary retention after semiclosed hemorrhoidectomy.

\section{Keywords: Risk factors; Hemorrhoidectomy; Spinal anesthesia; Urinary retention}

\section{INTRODUCTION}

Postoperative urinary retention is a complication that poses difficulties for both patients and colorectal surgeons. Its incidence ranges from $1 \%$ to $52 \%$, and it is the most common complication of anorectal surgery [1-3]. Among various anal surgeries, conventional hemorrhoidectomy has a higher incidence of urinary retention than fistulectomy and lateral sphincterotomy [1]. Although the incidence of urinary retention has decreased in stapled hemorrhoidectomy, it still shows a high incidence of over 15\% [4]. The

Received: Apr 27, 2021 - Revised: Jun 3, 2021 - Accepted: Jun 5, 2021 Correspondence to: Seok Gyu Song

Department of Surgery, Seoul Songdo Hospital, 72 Dasan-ro, Jung-gu, Seoul 04597, Korea

Tel: +82-2-2250-7368, Fax: +82-2-2233-8528

E-mail: vishnujin84@gmail.com

ORCID: https://orcid.org/0000-0001-6393-081X

(C) 2022 The Korean Society of Coloproctology

This is an open-access article distributed under the terms of the Creative Commons Attribution NonCommercial License (https://creativecommons.org/licenses/by-nc/4.0) which permits unrestricted noncommercial use, distribution, and reproduction in any medium, provided the original work is properly cited. exact etiology of urinary retention after hemorrhoidectomy remains unclear. However, some studies have shown that bladder overdistension may lead to a reduction in the contractile function of the detrusor muscle as well as chronic impairment of bladder emptying or atony [5]. Urinary retention is known to have multiple risk factors. The type of anesthesia $[6,7]$, postoperative pain [1], use of analgesics and opiates [8], volume of intravenous fluid [9], age [10], sex [11], and concomitant disease [12] have all been associated with the development of postoperative urinary retention.

Semiclosed hemorrhoidectomy is widely used in Korea and Japan to reduce postoperative pain, lower the risk of postoperative bleeding, and shorten the time required for the epithelization of the denuded anal canal [13]. Postoperative pain is an important contributing factor to urinary retention. Semiclosed hemorrhoidectomy that entails less postoperative pain may have a positive effect on urinary retention, but no research to date has evaluated this. Among anorectal diseases, hemorrhoids are the most common, and almost all hemorrhoidectomies are performed under spinal anesthesia. Therefore, it is essential to investigate the cause of urinary retention after hemorrhoidectomy. This study aimed to 
analyze the predictors that might contribute to urinary retention following semiclosed hemorrhoidectomy under spinal anesthesia.

\section{METHODS}

This study was approved by the Institutional Review Board of Seoul Songdo Hospital (No. 2020-011). The requirement for informed consent of patients was waived because of the retrospective nature of the study. This retrospective study enrolled a total of 2,176 consecutive patients with symptomatic grade III to IV hemorrhoids who underwent semiclosed hemorrhoidectomy between September 2018 and September 2019. All surgeries were performed under spinal anesthesia using bupivacaine by 5 colorectal surgeons with more than 5 years of experience. Benign comorbid diseases such as anal fistula and anal fissure and patients with inflammatory bowel disease were excluded. Demographic data, medical history, and surgical and follow-up details of the patients were recorded. Patients with a history of alcoholism included those who consumed alcohol at least once a week, and patients with a smoking history included all current smokers. Due to the characteristics of surgery for benign diseases, the underlying diseases including hypertension and diabetes mellitus were operated in a well-controlled state. All patients underwent anal manometry for the evaluation of anorectal physiology and for the measurement of maximal resting pressure (MRP) and maximal squeezing pressure (MSP). Patients were divided into $84 \mathrm{mmHg}$ for MRP, and $150 \mathrm{mmHg}$ for MSP according to the highest normal range. In addition, anal ultrasonography was performed to check for concomitant disease. The volume of intravenous fluid administered during operation did not exceed $500 \mathrm{~mL}$. For postoperative pain control, patient-controlled analgesia consisting of nefopam and pethidine was used. All patients were discharged from the hospital on postoperative day 2 and were admitted to the outpatient clinic 1 week postoperatively. In the outpatient clinic, the patients were orally administered analgesics for supplementary analgesia, when necessary, and orally administered laxative agents for constipation.

Urinary retention was defined as a case in which more than 300 $\mathrm{mL}$ of urine remained in the bladder, as determined using ultrasound performed after self-urination, and required the insertion of a Foley catheter or Nelaton catheter. We performed bladder ultrasound scans in all patients who could not urinate or had a sensation of urinary retention. Moreover, the patients were encouraged to take a sitz bath twice a day after surgery. The patients were divided into the retention group and the non-retention group according to the development of postoperative urinary retention. Patient demographics, physiological results, and operative findings were compared between the 2 groups. The risk factors for the development of urinary retention after semiclosed hemorrhoidectomy were analyzed.

Categorical variables are reported as numbers and percentages, and continuous variables are reported as means and standard de- viations. Significant differences between the types of bleeding were determined using the chi-square tests and the independent 2 -sample t-tests. Odds ratios (ORs) and 95\% confidence intervals (CIs) were estimated via logistic regression to determine the strength of association between the factors and urinary retention; the P-values of $<0.05$ were considered statistically significant. All statistical analyses were performed using IBM SPSS Statistics for Windows ver. 22.0 (IBM Corp., Armonk, NY, USA).

\section{RESULTS}

Among the 2,176 patients included in the study, 1,878 (86.3\%) had no postoperative urinary retention, whereas 298 (13.7\%) developed urinary retention after hemorrhoidectomy. Patient demographics are summarized in Table 1 . The percentage of males was significantly higher in the retention group than in the nonretention group ( $60.4 \%$ vs. $48.1 \%, \mathrm{P}=0.001)$. Significant differences were also observed between the retention and non-retention groups in age ( $53.23 \pm 13.76$ years vs. $47.71 \pm 14.30$ years, $\mathrm{P}=$ $0.001)$ and body mass index (BMI; $24.51 \pm 2.88$ vs. $23.71 \pm 3.52$ $\mathrm{kg} / \mathrm{m}^{2}, \mathrm{P}=0.001$ ). In particular, the proportion of elderly patients aged $\geq 65$ years ( $20.8 \%$ vs. $14.0 \%)$ and overweight patients with a BMI of $\geq 20 \mathrm{~kg} / \mathrm{m}^{2}$ (44.0\% vs. $32.1 \%$ ) was higher in the retention group than in the non-retention group. However, no significant differences were found between the retention and non-retention groups in the main symptom, drinking history, and smoking history. The incidence of hypertension $(28.2 \%$ vs. $18.2 \%, \mathrm{P}=0.001)$ was significantly higher in the retention group than in the nonretention group. Other than that, there was no difference between the 2 groups in the past medical history. The retention group had a significantly higher percentage of 4 or more than 4 resected hemorrhoids (43.0\% vs. $34.7 \%, \mathrm{P}=0.006$ ) and an American Society of Anesthesiologists (ASA) physical status (PS) classification of II or III ( $43.6 \%$ vs. $30.7 \%, \mathrm{P}=0.001)$. The use of analgesics ( $40.9 \%$ vs. $26.8 \%, \mathrm{P}=0.001)$ in supplementary medication was significantly difference in retention group (Table 1).

The laboratory test and anal manometry results are summarized in Table 2. The retention and non-retention groups differed in the proportion of patients with an MSP of $\geq 150 \mathrm{mmHg}(52.3 \%$ vs. $44.4 \%, \mathrm{P}=0.010$ ) in the anal manometry result. However, no difference was observed in the laboratory results and MRP between the 2 groups (Table 2).

\section{Predictors of postoperative urinary retention}

Table 3 shows the predictors for urinary retention after semiclosed hemorrhoidectomy. Univariate analysis showed that male sex, age, BMI, hypertension, high MSP, number of resected hemorrhoids, ASA PS classification II to III, and supplementary analgesics were significant risk factors for postoperative urinary retention. Results from the multivariate analysis showed that male sex, age, BMI, number of resected hemorrhoids, and supplementary analgesics were independent risk factors for postoperative urinary 
Table 1. Patients' demographics

\begin{tabular}{|c|c|c|c|}
\hline Demographic & Non-retention & Retention & P-value \\
\hline No. of patients & 1,878 & 298 & \\
\hline Male sex & $904(48.1)$ & $180(60.4)$ & 0.001 \\
\hline Age (yr) & $47.71 \pm 14.30$ & $53.23 \pm 13.76$ & 0.001 \\
\hline$\geq 65$ & $262(14.0)$ & $62(20.8)$ & \\
\hline$<65$ & $1,616(86.0)$ & $236(79.2)$ & \\
\hline Body mass index $\left(\mathrm{kg} / \mathrm{m}^{2}\right)$ & $23.71 \pm 3.52$ & $24.51 \pm 2.88$ & 0.001 \\
\hline$\geq 20$ & $602(32.1)$ & $131(44.0)$ & \\
\hline$<20$ & $1,276(67.9)$ & $167(56.0)$ & \\
\hline Main symptom & & & 0.514 \\
\hline Anal prolapse & $1,015(54.0)$ & $176(59.1)$ & \\
\hline Anal bleeding & $609(32.4)$ & $90(30.2)$ & \\
\hline Anal pain & $242(12.9)$ & $30(10.1)$ & \\
\hline Itching & $5(0.3)$ & $1(0.3)$ & \\
\hline Discharge & $7(0.4)$ & $1(0.3)$ & \\
\hline Drinking history & $932(49.6)$ & $142(47.7)$ & 0.534 \\
\hline Smoking history & $370(19.7)$ & $63(21.1)$ & 0.585 \\
\hline \multicolumn{4}{|l|}{ Past medical history } \\
\hline Hypertension & $342(18.2)$ & 84 (28.2) & 0.001 \\
\hline Diabetes mellitus & $91(4.8)$ & $19(6.4)$ & 0.256 \\
\hline Cardiovascular disease & $42(2.2)$ & $9(3.0)$ & 0.408 \\
\hline $\mathrm{BPH}$, incidence rate in male & $55 / 904(6.1)$ & 15/180 (8.3) & 0.074 \\
\hline Fatty liver & $59(3.1)$ & $5(1.7)$ & 0.198 \\
\hline Defecation time (min) & & & 0.306 \\
\hline$<5$ & $721(38.4)$ & $124(41.6)$ & \\
\hline$\geq 5$ & $1,157(61.6)$ & $174(58.4)$ & \\
\hline Hemorrhoid grade & & & 0.165 \\
\hline III & $1,344(71.6)$ & $225(75.5)$ & \\
\hline IV & $534(28.4)$ & $73(24.5)$ & \\
\hline No. of resected hemorrhoids & & & 0.006 \\
\hline$\geq 4$ & $651(34.7)$ & $128(43.0)$ & \\
\hline$<4$ & $1,227(65.3)$ & $170(57.0)$ & \\
\hline ASA PS classification & & & 0.001 \\
\hline 1 & $1,302(69.3)$ & $168(56.4)$ & \\
\hline$\|-1\|$ & $576(30.7)$ & $130(43.6)$ & \\
\hline \multicolumn{4}{|l|}{ Supplementary medication } \\
\hline Analgesics & $503(26.8)$ & $122(40.9)$ & 0.001 \\
\hline Laxative agent & $319(17.0)$ & $63(21.1)$ & 0.085 \\
\hline
\end{tabular}

Values are presented as number (\%) or mean \pm standard deviation.

$\mathrm{BPH}$, benign prostatic hyperplasia; ASA, American Society of Anesthesiologists; PS, physical status.

retention. The risk of urinary retention was 1.52 -fold higher in males than in females (95\% CI, 1.13-2.04; $\mathrm{P}=0.005), 1.62$-fold
Table 2. Laboratory test and anal manometry results

\begin{tabular}{lccc}
\hline Variable & $\begin{array}{c}\text { Non-retention } \\
(\mathrm{n}=1,878)\end{array}$ & $\begin{array}{c}\text { Retention } \\
(\mathrm{n}=298)\end{array}$ & P-value \\
\hline $\begin{array}{l}\text { Serum blood urea nitrogen } \\
\text { (mg/dL) }\end{array}$ & $13.21(10.6-15.4)$ & $13.84(11.5-16.2)$ & 0.244 \\
$\geq 20$ & $94(5.0)$ & $10(3.4)$ & \\
$<20$ & $1,784(95.0)$ & $288(96.6)$ & \\
Serum creatinine (mg/dL) & $0.77(0.6-0.9)$ & $0.81(0.7-0.9)$ & 0.091 \\
$\geq 1$ & $129(6.9)$ & $29(9.7)$ & \\
$<1$ & $1,749(93.1)$ & $269(90.3)$ & \\
Maximal resting pressure & $75.74 \pm 11.94$ & $75.33 \pm 12.27$ & 0.572 \\
(mmHg) & & & \\
$\geq 84$ & $493(26.3)$ & $83(27.9)$ & \\
$<84$ & $1,385(73.7)$ & $215(72.1)$ & \\
Maximal squeezing pressure & $151.20 \pm 57.28$ & $149.53 \pm 59.63$ & 0.010 \\
(mmHg) & & & \\
$\geq 150$ & $833(44.4)$ & $156(52.3)$ & \\
$<150$ & $1,045(55.6)$ & $142(47.7)$ & \\
\hline
\end{tabular}

Values are presented as median (interquartile range), number (\%), or mean \pm standard deviation.

higher in old age (95\% CI, 1.14-2.28; $\mathrm{P}=0.006)$, and 1.37 -fold higher in high BMI (95\% CI, 1.04-1.81; $\mathrm{P}=0.025)$. Patients with 4 or more than 4 resected hemorrhoids had a higher OR of 1.46 (95\% CI, 1.12-1.89; $\mathrm{P}=0.005$ ) than did patients with less than 4 resected hemorrhoids. Among the supplementary medication, patients who used analgesics had a higher OR of 2.06 (95\% CI, $1.57-2.68 ; \mathrm{P}=0.001$ ) than those who did not. However, no significant differences were found in the risk of urinary retention between patients with hypertension, high MSP, and ASA PS classification II to III (Table 3).

\section{DISCUSSION}

This retrospective study evaluated the predictors for postoperative urinary retention in patients who had undergone semiclosed hemorrhoidectomy. The reported incidence of urinary retention after anorectal surgery ranges from $1 \%$ to $52 \%[1-3,14]$. We found that the male sex, age, BMI, number of resected hemorrhoids, and supplementary analgesics were independent risk factors for postoperative urinary retention. Moreover, compared to the non-retention group, the retention group had a more significant hypertension, high MSP, and ASA PS classification II to III. The overall urinary retention rate of $13.7 \%$ determined in this study was within the reported range. The incidence of urinary retention differs according to the type of operation, type of anesthesia, exclusion criteria, and definition of urinary retention. In particular, the incidence is higher after hemorrhoidectomy. Zaheer et al. [1] reported that urinary retention developed in $16 \%$ of patients undergoing anorectal surgery. They also reported that the 
Annals of Predictors of postoperative urinary retention after semiclosed hemorrhoidectomy

Coloproctology Hong Yoon Jeong, et al.

Table 3. Predictors of urinary retention after semiclosed hemorrhoidectomy

\begin{tabular}{|c|c|c|c|c|}
\hline \multirow{2}{*}{ Variable } & \multicolumn{2}{|c|}{ Univariate analysis } & \multicolumn{2}{|c|}{ Multivariate analysis } \\
\hline & OR $(95 \% \mathrm{Cl})$ & P-value & OR $(95 \% \mathrm{Cl})$ & P-value \\
\hline Male sex & $1.64(1.28-2.11)$ & 0.001 & $1.52(1.13-2.04)$ & 0.005 \\
\hline Age, $\geq 65$ yr & $1.62(1.19-2.21)$ & 0.002 & $1.62(1.14-2.28)$ & 0.006 \\
\hline Body mass index, $\geq 20 \mathrm{~kg} / \mathrm{m}^{2}$ & $1.66(1.30-2.13)$ & 0.001 & $1.37(1.04-1.81)$ & 0.025 \\
\hline Drinking history & $0.92(0.72-1.18)$ & 0.526 & & \\
\hline Smoking history & $1.09(0.81-1.48)$ & 0.563 & & \\
\hline \multicolumn{5}{|l|}{ Past medical history } \\
\hline Hypertension & $1.76(1.34-2.33)$ & 0.001 & $1.11(0.77-1.60)$ & 0.570 \\
\hline Diabetes mellitus & $1.34(0.80-2.23)$ & 0.264 & & \\
\hline Cardiovascular disease & $1.36(0.66-2.83)$ & 0.408 & & \\
\hline Benign prostatic hyperplasia & $1.76(0.98-3.15)$ & 0.059 & & \\
\hline Fatty liver & $0.53(0.21-1.32)$ & 0.172 & & \\
\hline Defecation time, $\geq 5$ min & $0.87(0.68-1.12$ & 0.290 & & \\
\hline \multicolumn{5}{|l|}{ Hemorrhoid grade } \\
\hline III & 1 & & & \\
\hline IV & $0.82(0.62-1.08)$ & 0.160 & & \\
\hline \multicolumn{5}{|l|}{ Laboratory result } \\
\hline Blood urea nitrogen, $\geq 20 \mathrm{mg} / \mathrm{dL}$ & $0.66(0.34-1.28)$ & 0.218 & & \\
\hline Serum creatinine, $\geq 1 \mathrm{mg} / \mathrm{dL}$ & $1.46(0.96-2.23)$ & 0.078 & & \\
\hline \multicolumn{5}{|l|}{ Physiological results } \\
\hline MRP, $\geq 84 \mathrm{mmHg}$ & $1.09(0.83-1.43)$ & 0.561 & & \\
\hline MSP, $\geq 150 \mathrm{mmHg}$ & $1.38(1.08-1.76)$ & 0.010 & $1.10(0.83-1.45)$ & 0.501 \\
\hline \multicolumn{5}{|l|}{ No. of resected hemorrhoids } \\
\hline$<4$ & 1 & & & \\
\hline$\geq 4$ & $1.42(1.11-1.82)$ & 0.006 & $1.46(1.12-1.89)$ & 0.005 \\
\hline \multicolumn{5}{|l|}{ ASA PS classification } \\
\hline 1 & 1 & & & \\
\hline$\|-\| \|$ & $1.75(1.36-2.24)$ & 0.001 & $1.29(0.95-1.76)$ & 0.101 \\
\hline \multicolumn{5}{|l|}{ Supplementary medication } \\
\hline Analgesics & $1.90(1.47-2.44)$ & 0.001 & $2.06(1.57-2.68)$ & 0.001 \\
\hline Laxative agent & $1.31(0.97-1.77)$ & 0.081 & & \\
\hline
\end{tabular}

OR, odds ratio; $\mathrm{Cl}$, confidence interval; MRP, maximal resting pressure; MSP, maximal squeezing pressure; ASA, American Society of Anesthesiologists; PS, physical status.

incidence was $4 \%$ after lateral sphincterotomy and $2 \%$ after fistulotomy, but $34 \%$ after hemorrhoidectomy. To reduce urinary retention, our hospital employs measures such as oral analgesia, hot pack application, and ambulation for patients who cannot void 6 hours after undergoing hemorrhoidectomy. In addition, we limit intravenous fluid administration to $<500 \mathrm{~mL}$ during operation as part of fluid restriction. Under spinal anesthesia, a large volume of intravenous fluid can cause irreversible detrusor muscle dysfunction because of impaired bladder sensation and inhibition of the bladder reflex [15]. Bailey and Ferguson [2] reported a reduction in postoperative urinary retention from $15 \%$ to $4 \%$ in patients for whom both intravenous and oral fluids were restricted. The above efforts have lowered the incidence of urinary retention after hemorrhoidectomy.

The definition of postoperative urinary retention is controversial. In previous studies, urinary retention was defined as the need for catheterization within 24 hours after the surgery $[4,16]$. In our study, urinary retention was defined based on the need for catheterization as well as postvoid residual urine evaluation. Bladder ultrasound scan is a simple, noninvasive, and cost-effective 
method to measure the volume of urine within the bladder. To date, there is no consensus on the cutoff volume to define urinary retention. Several studies have defined urinary retention as a postvoid residual urine volume greater than $150 \mathrm{~mL}$ or $200 \mathrm{~mL}$ $[17,18]$. The American Urological Association has recommended considering a value greater than $300 \mathrm{~mL}$ as urinary retention. The present study used this criterion to define urinary retention [19].

The cause of urinary retention after hemorrhoidectomy remains unclear. However, previous studies have suggested that bladder outlet obstruction or inhibited bladder contraction is the cause of retention [20, 21]. Barone and Cummings [20] suggested that retention following anorectal surgery is due to bladder outlet obstruction. Sympathetic nerve discharge causes catecholamine release and alpha adrenergic-mediated contraction of the bladder neck, resulting in functional obstruction of the bladder outlet. Our analysis showed that the male sex was an independent risk factor for urinary retention. However, previous studies have reported conflicting findings. While some studies reported that male sex was a risk factor $[1,22]$, another study reported that female sex was a risk factor [20]. Furthermore, other studies reported no statistical difference in the incidence of urinary retention between the sexes $[14,23,24]$. Naturally, male sex is a risk factor because $53 \%$ of urinary retention is caused by benign prostatic hyperplasia (BPH) [25]. Interestingly, urinary retention is quite common among patients with $\mathrm{BPH}$, but the difference is not statistically significant. This could be because BPH is the most common cause of obstructive urinary retention [25]. In our results, the incidence rate of $\mathrm{BPH}$ in men $(8.3 \%$ vs. $6.1 \%, \mathrm{P}=0.074)$ was higher in the retention group. The reason for these results is that most of the patients with $\mathrm{BPH}$ included in this study were on alpha-blockers. In addition, urinary retention was significantly higher in patients with hypertension. A previous study also reported that the use of beta-blockers was associated with an increased risk of postoperative urinary retention [26]. Although an analysis of drugs administered for hypertension was not conducted in this study, the use of beta-blockers among patients with hypertension might account for this result.

Pompeius [21] suggested that inhibition of the detrusor muscle is a result of a reflex involving the afferent fibers of the pudendal nerve and efferent pelvic sympathetic nerves. Perianal pain and overdistention of the bladder trigger this reflex. Toyonaga et al. [16] suggested that urinary retention may be related to the degree of pain. Our data showed that urinary retention was frequently observed in patients who underwent the removal of more hemorrhoid piles. Removing multiple hemorrhoid piles would have widened the wound and increased the postoperative pain. Although the evaluation of postoperative pain in this study was limited, the number of resected hemorrhoids and supplementary analgesics indirectly showed that pain was the cause of urinary retention. In particular, 2.06-fold more urinary retention occurred in patients who used supplemental analgesics, suggesting that postoperative pain is a risk factor for urinary retention. We used semiclosed hemorrhoidectomy to reduce postoperative pain, and this was one of the reasons for the lower incidence of urinary retention in our study than in other studies.

Our study also showed that old age is an independent risk factor for urinary retention. Studies have reported that detrusor function deteriorates progressively and that bladder sensation declines with advancing age [27]. Furthermore, the elderly may be more susceptible to the negative urodynamic effects of anesthetic agents because many of these drugs have a prolonged duration of action in them [16]. Therefore, in elderly patients, proper pain control will be useful in reducing postoperative urinary retention. Some studies have suggested that diabetes mellitus is also a risk factor for urinary retention. Approximately $25 \%$ to $60 \%$ of men and women with diabetes mellitus will develop diabetic cystopathy, which can lead to detrusor underactivity and urinary retention [28]. In our study, patients with diabetes mellitus as a comorbidity experienced more urinary retention, but the difference was not statistically significant. Nevertheless, this finding has limited applicability because of the dearth of investigations on the prevalence of urinary retention in patients with diabetes mellitus.

The prevalence of urinary retention is high among patients with morbid obesity, ranging from $32 \%$ to $66 \%$ [29]. Several factors, such as increased intra-abdominal pressure, stress on the pelvic floor, and neuromuscular dysfunction of the genitourinary tract, have been proposed to be important [30]. Although there are no studies on the incidence of urinary retention after hemorrhoidectomy in patients with obesity, the incidence is considered to be similar. Moreover, BMI and waist circumference have all been positively associated with prostate volume in multiple study populations [31]. We found that high BMI was an independent risk factor for urinary retention after hemorrhoidectomy. Owing to the characteristics of East Asian races, there were few patients with severe obesity. Therefore, the high BMI standard was set to $\geq 20 \mathrm{~kg} / \mathrm{m}^{2}$. For a greater number of patients, the analysis based on a higher BMI is expected to obtain a higher OR.

A limitation of this study is the use of a cross-sectional, retrospective, and non-randomized analysis. Moreover, as our institution is a specialized colorectal hospital, the diagnosis of BPH was based only on medical history. In a future study, we intend to measure and compare prostate size with urinary retention for quantitative evaluation of $\mathrm{BPH}$. Another limitation is the lack of data on postoperative pain. Several previous studies have reported that postoperative urinary retention is closely associated with postoperative pain score [32]. In our data, there were no recorded pain scores immediately after surgery and when admitted to the outpatients clinic, so it was not included in this analysis. In future studies, it is necessary to analyze the postoperative pain. However, as an indirect indicator of pain, the use of supplemental analgesics and the number of resected hemorrhoids reflected postoperative pain in this study. The strength of this study is that all patients underwent semiclosed hemorrhoidectomy under spinal anesthesia. In addition, experienced surgeons and more than 2,000 patients 
were included in this study. Our findings also highlight the need for further research on treatment methods that can reduce urinary retention after hemorrhoidectomy.

In conclusion, male sex, age, BMI, hypertension, high MSP, number of resected hemorrhoids, ASA PS classification II to III, and supplementary analgesics are predictors for urinary retention. In particular, male sex, age, high BMI, number of resected hemorrhoids, and supplementary analgesics are independent risk factors for urinary retention after semiclosed hemorrhoidectomy. Therefore, surgeons should always be mindful of urinary retention after hemorrhoidectomy when performing surgery in patients with these predictors.

\section{CONFLICT OF INTEREST}

No potential conflicts of interest relevant to this article were reported.

\section{REFERENCES}

1. Zaheer S, Reilly WT, Pemberton JH, Ilstrup D. Urinary retention after operations for benign anorectal diseases. Dis Colon Rectum 1998;41:696-704.

2. Bailey HR, Ferguson JA. Prevention of urinary retention by fluid restriction following anorectal operations. Dis Colon Rectum 1976;19:250-2.

3. Hoff SD, Bailey HR, Butts DR, Max E, Smith KW, Zamora LF, et al. Ambulatory surgical hemorrhoidectomy: a solution to postoperative urinary retention? Dis Colon Rectum 1994;37:1242-4.

4. Chik B, Law WL, Choi HK. Urinary retention after haemorrhoidectomy: impact of stapled haemorrhoidectomy. Asian J Surg 2006;29:233-7.

5. Hinman F. Editorial: postoperative overdistention of the bladder. Surg Gynecol Obstet 1976;142:901-2.

6. Darrah DM, Griebling TL, Silverstein JH. Postoperative urinary retention. Anesthesiol Clin 2009;27:465-84.

7. Lamonerie L, Marret E, Deleuze A, Lembert N, Dupont M, Bonnet F. Prevalence of postoperative bladder distension and urinary retention detected by ultrasound measurement. Br J Anaesth 2004;92:544-6.

8. Kumar P, Mannan K, Chowdhury AM, Kong KC, Pati J. Urinary retention and the role of indwelling catheterization following total knee arthroplasty. Int Braz J Urol 2006;32:31-4.

9. Keita H, Diouf E, Tubach F, Brouwer T, Dahmani S, Mantz J, et al. Predictive factors of early postoperative urinary retention in the postanesthesia care unit. Anesth Analg 2005;101:592-6.

10. O'Riordan JA, Hopkins PM, Ravenscroft A, Stevens JD. Patientcontrolled analgesia and urinary retention following lower limb joint replacement: prospective audit and logistic regression analysis. Eur J Anaesthesiol 2000;17:431-5.

11. Sarasin SM, Walton MJ, Singh HP, Clark DI. Can a urinary tract symptom score predict the development of postoperative urinary retention in patients undergoing lower limb arthroplasty under spinal anaesthesia? A prospective study. Ann R Coll Surg Engl 2006;88:394-8.

12. Izard JP, Sowery RD, Jaeger MT, Siemens DR. Parameters affecting urologic complications after major joint replacement surgery. Can J Urol 2006;13:3158-63.

13. Takano M. Anoderm-preserving hemorrhoidectomy. Dis Colon Rectum 1980;23:544-9.

14. Petros JG, Bradley TM. Factors influencing postoperative urinary retention in patients undergoing surgery for benign anorectal disease. Am J Surg 1990;159:374-6.

15. Tammela T. Postoperative urinary retention: why the patient cannot void. Scand J Urol Nephrol Suppl 1995;175:75-7.

16. Toyonaga T, Matsushima M, Sogawa N, Jiang SF, Matsumura N, Shimojima $\mathrm{Y}$, et al. Postoperative urinary retention after surgery for benign anorectal disease: potential risk factors and strategy for prevention. Int J Colorectal Dis 2006;21:676-82.

17. Kin C, Rhoads KF, Jalali M, Shelton AA, Welton ML. Predictors of postoperative urinary retention after colorectal surgery. Dis Colon Rectum 2013;56:738-46.

18. Revicky V, Mukhopadhyay S, de Boer F, Morris EP. Obesity and the incidence of bladder injury and urinary retention following tension-free vaginal tape procedure: retrospective cohort study. Obstet Gynecol Int 2011;2011:746393.

19. Stoffel JT, Peterson AC, Sandhu JS, Suskind AM, Wei JT, Lightner DJ. AUA White paper on nonneurogenic chronic urinary retention: consensus definition, treatment algorithm, and outcome end points. J Urol 2017;198:153-60.

20. Barone JG, Cummings KB. Etiology of acute urinary retention following benign anorectal surgery. Am Surg 1994;60:210-1.

21. Pompeius R. Detrusor inhibition induced from anal region in man. Acta Chir Scand Suppl 1966;361:1-54.

22. Crystal RF, Hopping RA. Early postoperative complications of anorectal surgery. Dis Colon Rectum 1974;17:336-41.

23. Bowers FJ, Hartmann R, Khanduja KS, Hardy TG Jr, Aguilar PS, Stewart WR. Urecholine prophylaxis for urinary retention in anorectal surgery. Dis Colon Rectum 1987;30:41-2.

24. Stallard S, Prescott S. Postoperative urinary retention in general surgical patients. Br J Surg 1988;75:1141-3.

25. Choong S, Emberton M. Acute urinary retention. BJU Int 2000; 85:186-201.

26. Boulis NM, Mian FS, Rodriguez D, Cho E, Hoff JT. Urinary retention following routine neurosurgical spine procedures. Surg Neurol 2001;55:23-7.

27. Pfisterer MH, Griffiths DJ, Schaefer W, Resnick NM. The effect of age on lower urinary tract function: a study in women. J Am Geriatr Soc 2006;54:405-12.

28. Kebapci N, Yenilmez A, Efe B, Entok E, Demirustu C. Bladder dysfunction in type 2 diabetic patients. Neurourol Urodyn 2007; 26:814-9.

29. Vella VL, Jaffe W, Lidicker J, Meilahn J, Dandolu V. Prevalence of urinary symptoms in morbidly obese women and changes after 
bariatric surgery. J Reprod Med 2009;54:597-602.

30. Laungani RG, Seleno N, Carlin AM. Effect of laparoscopic gastric bypass surgery on urinary incontinence in morbidly obese women. Surg Obes Relat Dis 2009;5:334-8.

31. Parsons JK. Modifiable risk factors for benign prostatic hyperpla- sia and lower urinary tract symptoms: new approaches to old problems. J Urol 2007;178:395-401.

32. Qi-Ming X, Jue-Ying X, Ben-Hui C, Jing W, Ning L. Risk factors for postoperative retention after hemorrhoidectomy: a cohort study. Gastroenterol Nurs 2015;38:464-8. 